\title{
Study on Processing Technology and Quality of Moringa oleifera leaves with Y - Aminobutyric Acid
}

\author{
ZHOU Wei ${ }^{1}$, LI Qian ${ }^{1,2}$, GUO Changqing ${ }^{3}$, CAO Yupo ${ }^{1}$, PENG Shaodan ${ }^{1}$, JING Wei $^{1}$, LI Jihua ${ }^{1},{ }^{*}$ \\ ${ }^{1}$ Agricultural Products Processing Research Institute, Chinese Academy of Tropical Agricultural Sciences, Zhanjiang, Guangdong, 524001, \\ China \\ ${ }^{2}$ College of Food Science and Technology, GuangDong Ocean University, Zhanjiang, Guangdong, 524088, China; \\ ${ }^{3}$ Henan Gold Moringa Biotechnology Co., Ltd., Hebi, Henan, 458000, China
}

\begin{abstract}
In order to obtain the high level of $\gamma$-aminobutyric acid Moringa oleifera leaves, Use 7\% sodium glutamate solution to soak the fresh Moringa oleifera leaves, study effect of different treatment times and three different drying methods ( hot air drying, vacuum freeze drying, shadow drying ) on the formation of $\gamma$-aminobutyric acid and quality (total flavonoids, soluble sugar, amino acids, polyphenols, color)of dried Moringa oleifera leaves. The results indicated that shadow-dried Moringa oleifera leaves had the hightest retention of $\gamma$-aminobutyric acid, but its browning degree were not preferable, soluble sugar was damaged gravely, and its vulnerable to weather conditions. Vacuum freeze dried Moringa oleifera leaves had the hightest retention of flavonoids, polyphenols and amino acids. The $\gamma$-aminobutyric acid content of Vacuum freeze dried and hot air dried Moringa oleifera leaves had no much difference. Hot air dried Moringa oleifera leaves browning degree were preferable, it's had an moderate content of soluble sugar and amino acids, the short drying time is characteristics of this drying method.with the treatment time increased, the content of $\gamma$ aminobutyric acid and amino acids content first increased and then decreased. Flavonoids and polyphenols content first decreased and then increased. Soluble sugar content decreased. In summary, after soaking with $7 \%$ sodium glutamate solution for $10 \mathrm{~h}$, then dried by hot air drying $\left(\right.$ drying temperature of $\left.60^{\circ} \mathrm{C}\right)$, was the most suitable way for industrial production of the high level of $\gamma$-aminobutyric acid Moringa oleifera leaves.
\end{abstract}

\section{Introduction}

Moringa oleifera Lam, is a species belonging to the Moringa Branch and Moringaceae family, widely distributed intropical and subtropical regions of Asia, Africa ${ }^{[1]}$.The whole plant has edible value and can be useful $^{[2]}$.Its roots, stems, leaves, flowers, seeds, branches and barks are rich in nutrients and medicinal ingredients ${ }^{[3]}$. Studies have shown that Moringa leaves are rich in potassium, calcium, phosphorus, iron, and essential amino acids, and having antioxidant activity of vitamin C, flavonoids and polyphenols substances ${ }^{[3]}$. Moringa has antioxidant, anti-cancer, hypoglycemic and lipid-lowering and other biological activities ${ }^{[4-7]}$.

$\gamma$-aminobutyric acid (GABA) is a non protein natural amino acid. It has many physiological activities, such as lowering blood pressure, anti-aging and anti-anxiety ${ }^{[8]}$. GABA in plants can be formed by L-glutamic acid or its salts under irreversible $\alpha$ decarboxylation catalyzed by glutamate decarboxylase (GAD) ${ }^{[9]} . \gamma$-aminobutyric acid can be biosynthesized by GAD, but with the increase of age and mental stress, the accumulation of GABA in the human body is very difficult. Although GABA is widely distributed, the content of GABA is very small ${ }^{[10]}$. With the immersion of exogenous amino acids as a enrichment technique,GABA biosynthetic substrates can be added not only to form hypoxic environments, to activate GAD activity, but also to promote the synthesis of large amounts of GABA [11]. Many scholars have applied exogenous amino acid soaking technology to natural products such as tea, soybean and brown rice ${ }^{[12-13]}$, but there are few reports on improving the content of GABA in Moringa oleifera ${ }^{[14-}$ ${ }^{15]}$. Zhao et al. ${ }^{[14]}$ and others used glutamic acid as the soaking solution to increase the content of GABA in the leaves of Moringa, but did not study the effect of soaking on the quality components of Moringa oleifera. The research work needs to be further studied.

Drying is the last step in the processing of GABA Moringa leaves. Drying can reduce the moisture content of the material, effectively hinder the breeding of microorganisms within the material and the chemical reaction of water as the medium, thus significantly extending the shelf life of food and reducing the cost of storage and transportation ${ }^{[16]}$. However, different drying methods have a great influence on the quality components in the material, so it is necessary to use a suitable drying method.

Therefore, the experiment was carried out by drying at $60{ }^{\circ} \mathrm{C}$ oven, vacuum freeze-drying and natural drying to study the effects of three kinds of drying methods on GABA content and quality components of Moringa oleifera leaves, and to determine the best drying method. 
Moringa oleifera GABA enrichment process test in Postharvest Moringa leaf as raw material, with 7\% sodium glutamate (MSG) solution (D) as a solution, the best drying method was used to dry the fresh mulberry leaves after soaking. The effects of different immersion times on GABA content and quality components in Moringa lanceolata were studied. The results showed that Moringa oleifera leaves was rich in GABA, which was developed for the high content of $\gamma$-aminobutyric acid and other products lay the foundation.

\section{Materials and method}

\subsection{Plant material}

GABA Moringa leaves: fresh leaves of Moringa oleifera were bought from Henan gold M.oleifera Biological Technology Co. Ltd.

\subsection{Chemical and instruments}

2,4-dinitrofluorobenzen; $\gamma$-aminobutyric acid; glutamic acid; rutin gallate; Fulin phenol; chlorination Aluminum; sodium bicarbonate; sodium carbonate; potassium dihydrogen phosphate; sodium hydroxide; anhydrous sodium acetate; glacial acetic acid; N, Ndimethylformamide; anthrone and glucose.

High purity liquid chromatography Japan Shimadzu Corporation; UV1780 UV spectrophotometer Japan Shimadzu Corporation; ALPHA2-4 experimental freeze dryer Germany CHRIST company; DHG-9426A oven Shanghai Jinghong Experimental Equipment Co., Ltd; HH-4 constant temperature water bath Pots Jintan Guowang Experimental Instrument Factory; MilliQlntegral pure water meter France Mi Mi Bo company; pH meter METTLER TOLEDO company; color measuring instrument

\subsection{Effects of different drying methods on GABA content and color of tea in Moringa oleifera leaves}

The 4 groups of Moringa fresh leaves, each $50 \mathrm{~g}$, were placed in the white porcelain plate, respectively by $60{ }^{\circ} \mathrm{C}$ (A) drying and vacuum freeze drying (B) and natural dried (C) drying the material, the final moisture content control in $5 \%$.

\subsection{Effects of dipping treatment on GABA content of fresh leaves of Moringa oleifera and nutritional components}

The 7 groups of fresh leaves of Moringa, 20g each, were immersed in $300 \mathrm{ml} \%$ MSG solution (D), plastic film sealed bottle, all operations were carried out at room temperature, soaking time were $0 \mathrm{~h}, 2 \mathrm{~h}, 4 \mathrm{~h}, 6 \mathrm{~h}, 8 \mathrm{~h}, 10 \mathrm{~h}, 12 \mathrm{~h}$, remove the water after washing, steaming to about $30 \mathrm{~s} .60{ }^{\circ} \mathrm{C}$ blast drying box drying to a moisture content of around $5 \%$, and then analyze the content of GABA after treatment of Moringa oleifera and the main quality components, using pure water as the blank control $(\mathrm{CK})$, each sample was repeated 3 times.

\subsection{Determination of indicators and methods}

Flavonoids, Polyphenol Determination With reference to the method of Vongsak ${ }^{[15]}$, the total amount of free amino acids is determined by reference to GB / T 8314-2013, the determination of soluble total sugar content by reference to the method of Wei Wei ${ }^{[16]}$ et al.

GABA content of the method of determination: mobile phase: A $0.05 \mathrm{~mol} / \mathrm{L}$ sodium acetate buffer ( $\mathrm{pH} 6.8$, containing $1 \% \mathrm{~N}, \mathrm{~N}$-dimethyl formamide); B $50 \%$ acetonitrile aqueous solution (V/V) Gradient elution, flow rate $0.8 \mathrm{ml} / \mathrm{min}$, column temperature $27^{\circ} \mathrm{C}$, wavelength $360 \mathrm{~nm}$.

Amino acid pre-column derivatization: take the sample solution $1.0 \mathrm{ml}$ placed in $10 \mathrm{ml}$ volumetric flask, add $0.5 \mathrm{~mol} / \mathrm{L}(\mathrm{pH} 9.0)$ sodium bicarbonate $1.0 \mathrm{ml}$, mixed, and then add $1 \%$ 2,4-dinitrofluorobenzene $0.25 \mathrm{ml}$, the vortex oscillation uniform, in $60{ }^{\circ} \mathrm{C}$ water bath for $60 \mathrm{~min}$, remove and cool to room temperature, with $0.01 \mathrm{~mol} / \mathrm{L}(\mathrm{pH} 7.0)$ potassium dihydrogen phosphate buffer solution to the volume, with $0.45 \mu \mathrm{m}$ filter, machine.

\subsection{Experimental data processing}

The data were processed and analyzed by Origin 8.5 and SPSS 22.0 software. The data were analyzed by one-way ANOVA $(\mathrm{P}<0.05)$. All the experiments were repeated three times. The experimental results were expressed as mean \pm standard deviation.

\section{Results and discussion}

3.1 Effects of Different Drying Methods on GABA Content and Quality Components of Moringa oleifera Leaves

\subsubsection{Effects of three drying methods on GABA} content in Moringa oleifera leaves 


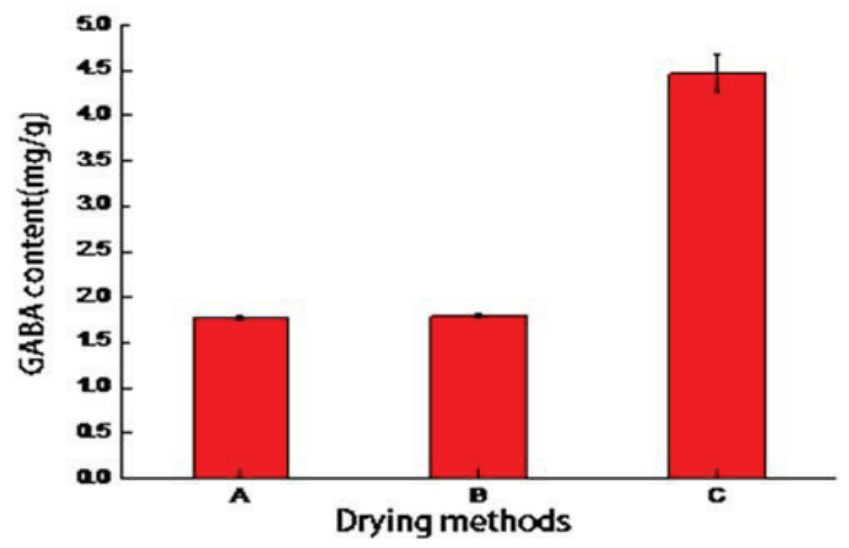

Fig.1 Effects of different drying methods on GABA content of Moringa oleifera leaves

The results of GABA content of the three kinds of drying methods are as follows: natural dryness (C)> vacuum freeze drying(B)> $60{ }^{\circ} \mathrm{C}$ blast drying(A)(Fig.1). Natural dryness is significantly higher than the other two drying methods, it may be because at room temperature during the drying process, protein hydrolase and glutamate decarboxylase and other related enzymes in an active state, glutamic acid and other amino acid content increased, thereby enhancing the Moringa oleifera Leaves GABA content.

\subsubsection{Effects of Four Drying Methods on Flavonoids} and Polyphenol Contents in Moringa oleifera Leaves

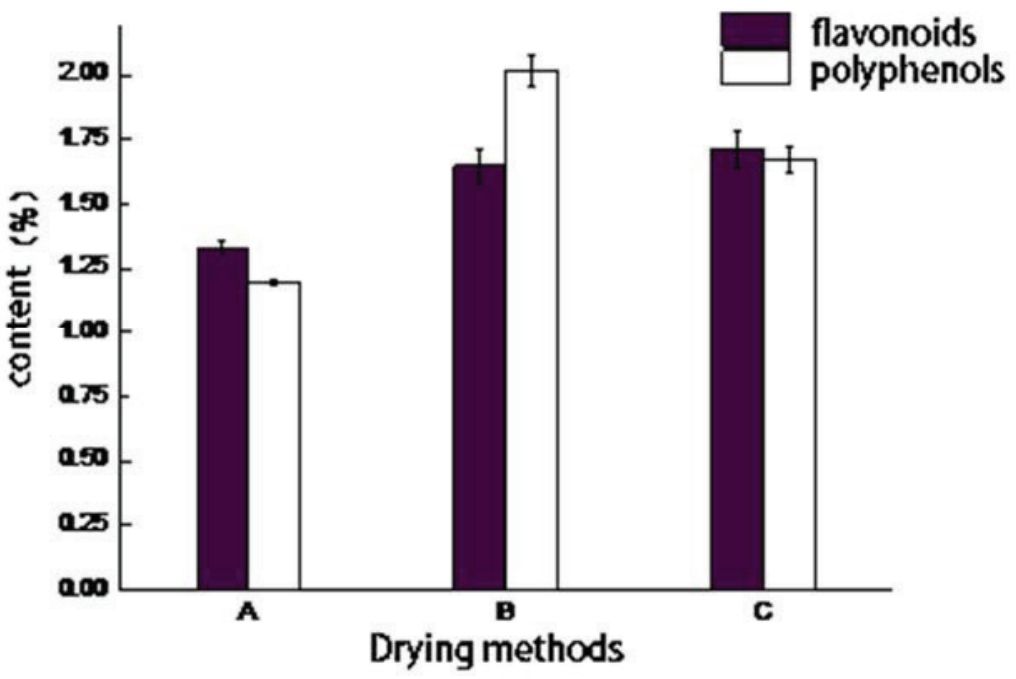

Fig.2 Effects of different drying methods on flavonoids and polyphenols content of Moringa oleifera leaves

The contents of the flavonoids from the highest to the lowest were as follows: natural drying $(\mathrm{C})$ and vacuum freeze drying(B), drying at $60{ }^{\circ} \mathrm{C}(\mathrm{A})$, The polyphenol content from high to low are: vacuum freeze drying(B), natural drying(C), drying at $60{ }^{\circ} \mathrm{C}$ (A) (Fig.2). Vacuum Freeze Drying Moringa leaves are in a vacuum state to avoid contact with oxygen, thereby reducing the loss of oxidation. Compared with B treatment, the content of polyphenol in $\mathrm{C}$ was lower, the content of flavonoids was almost the same, which may be due to the enzymatic browning of phenolic substances during the long period of drying.

\subsubsection{Effects of three drying methods on free amino} acid and soluble total sugar content in Moringa oleifera leaves 


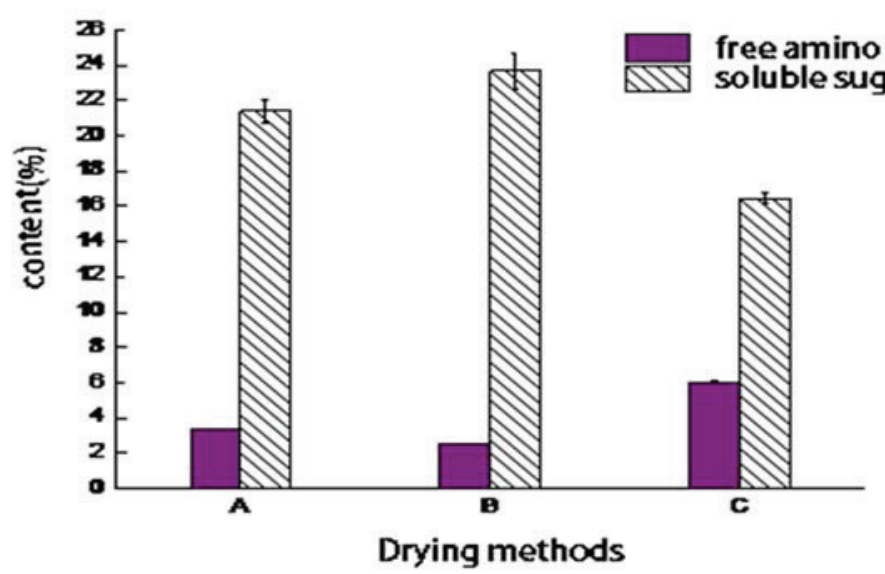

Fig.3 Effects of different drying methods on soluble sugar and amino acids content of Moringa oleifera leaves

The Moringa fresh leaves after different drying, the soluble sugar content from high to low order: vacuum freeze drying(B), $60^{\circ} \mathrm{C}$ blast drying(A), natural dry(C). The free amino acid content from high to low order: natural dry $(\mathrm{C})$, $60{ }^{\circ} \mathrm{C}$ blast drying(A), vacuum freezedrying(B)(Fig.3). The free amino acids of natural dry treatment was significantly higher than that of blast drying and vacuum freeze-drying treatments, which may be related to the high content of GABA in the treatment of Moringa oleifera.The free amino acid content of vacuum freeze-drying treatment is the lowest, probably because of low temperature and low oxygen conditions, the relevant enzyme activity is inhibited, the protein is not easily hydrolyzed into amino acids. The total soluble sugar content of B was higher than that of $\mathrm{A}$ group and $\mathrm{C}$ group, the reason may be in low temperature and hypoxia conditions, total soluble sugar not prone to oxidation and Maillard reaction, and the total soluble sugars and effective retention.

\subsubsection{The effect of three kinds of drying methods on the color of the tea leaves of Moringa oleifera}

Table.1 Effects of different drying methods on the Moringa oleifera leaves tea color

\begin{tabular}{ccccc}
\hline \multirow{2}{*}{ Drying methods } & \multicolumn{4}{c}{ Chromatic aberration } \\
\cline { 2 - 5 } & $\mathrm{L}^{*}$ & $\mathrm{a}^{*}$ & $\mathrm{~b}^{*}$ & $-\mathrm{a}^{*} / \mathrm{b}^{*}$ \\
\hline $\mathrm{A}$ & 88.82 & -4.04 & 34.75 & 0.116 \\
$\mathrm{~B}$ & 88.98 & -2.28 & 25.88 & 0.088 \\
C & 86.01 & -5.67 & 50.46 & 0.112 \\
& & & \\
\hline
\end{tabular}

For Moringa oleifera leaves, the bigger the $\mathrm{L}$ value, tea color brightness is better; the bigger the -a value, the soup color is green. The greater the $b$ value, the more yellow the color of the soup; The smaller the $-\mathrm{a} / \mathrm{b}$ value is, the higher the color of the soup is and the darker it is. The Moringa oleifera leaves prepared by three kinds of drying methods were soaked in boiling water, and the soup color was quantitatively analyzed. From the experimental results in Table 1, we can see that in the three drying methods, the brightness of tea is the best in B way, the $\mathrm{L} *$ is 88.98 , followed by $\mathrm{A}$ and $\mathrm{C}, \mathrm{L} *$ values are 88.82 and 86.01 respectively. soup color of B way brightness slightly higher than the A way, but its-a* / b * value is less than A way and $\mathrm{B}$ way, the highest degree of tea color and the darkest.

\subsection{Effects of $7 \%$ MSG solution immersion on GABA content and quality components of fresh sprouts}

\subsubsection{Effects of different immersion time on GABA content in Moringa oleifera leaves}




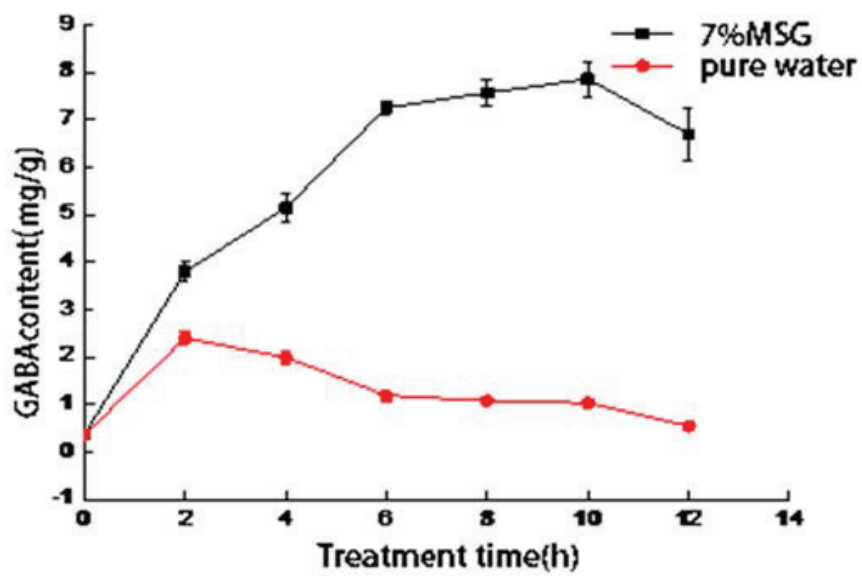

Fig.4 Effects of different treatment time on GABA content of Moringa oleifera leaves

The content of GABA in the leaves of 7\% MSG solution was significantly higher than that in the pure water treatment group (blank control) within $2 \mathrm{~h} \sim 12 \mathrm{~h}$, and the GABA content reached the maximum at $10 \mathrm{~h}$ in the $7 \%$ MSG solution, the blank control group reached the maximum at $2 \mathrm{~h}$ (Fig.4). With the prolongation of immersion time, the contents of GABA in the two treatment groups increased first and then decreased, which probably due to the excessive accumulation of GABA in
Moringa oleifera leaves, leading to the activation of GABA-degrading enzymes such as succinic acid and aldehyde dehydrogenase [18], thereby producing antirepressive effects on GABA synthesis.

\subsubsection{Effects of Different Immersion Time on Contents of Polyphenols and Flavonoids in Moringa oleifera Leave}
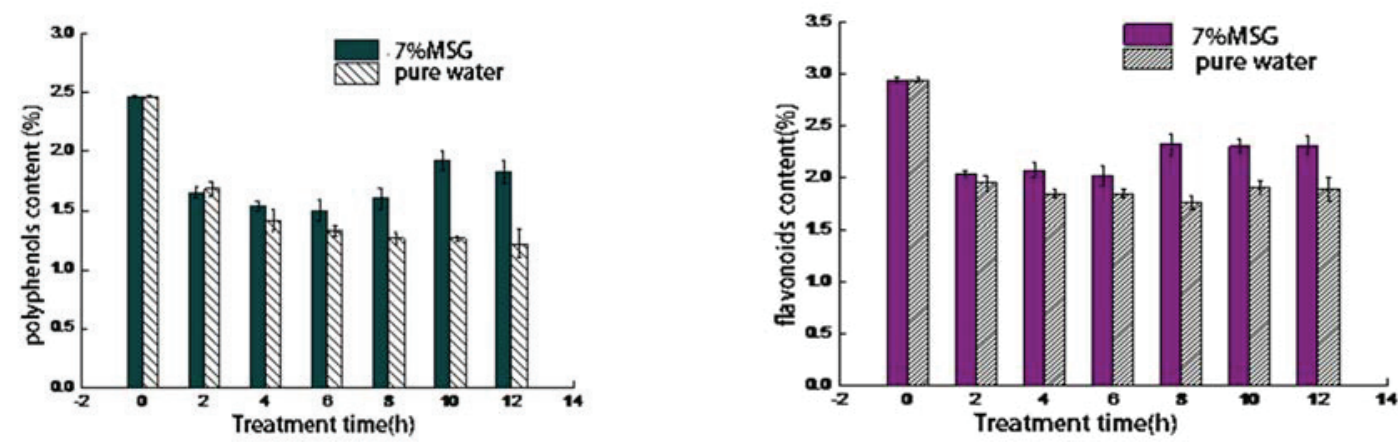

Fig.5 Effects of different treatment time on polyphenols content of Moringa oleifera leaves Fig.6 Effects of different treatment time on flavonoids content of Moringa oleifera leaves

The content of polyphenols and flavonoids in the D treatment group was higher than that in CK treatment group under the same impregnation time(Fig.5 and Fig.6). With the prolongation of immersion time, the content of polyphenols and flavonoids in $\mathrm{CK}$ treatment group decreased gradually, which may be due to phenolic substances react with the reaction of oxidation and hydrolysis under the action of enzyme. The content of polyphenols and flavonoids in D treatment group decreased first and then increased, but the content of polyphenols and flavonoids varied little in the range of $1.65 \%$ $\sim 1.82 \%$ and $2.01 \% \sim 2.31$ respectively in the range of $2 \sim$ $12 \%$, Were lower than $0 \mathrm{~h}$ treatment group. After $8 \mathrm{~h}$ treatment, the content of flavonoids and polyphenols increased slightly, which may be due to the excessive accumulation of GABA content of Moringa oleifera to promote the metabolism of phenolic substances, its increase in the amount of its own oxidation and dissolved loss, and thus the content of flavonoids and polyphenols increased, which remains to be further explored.

\subsubsection{Effects of different immersion time on soluble total sugar and free amino acids in fresh Moringa oleifera leaves}



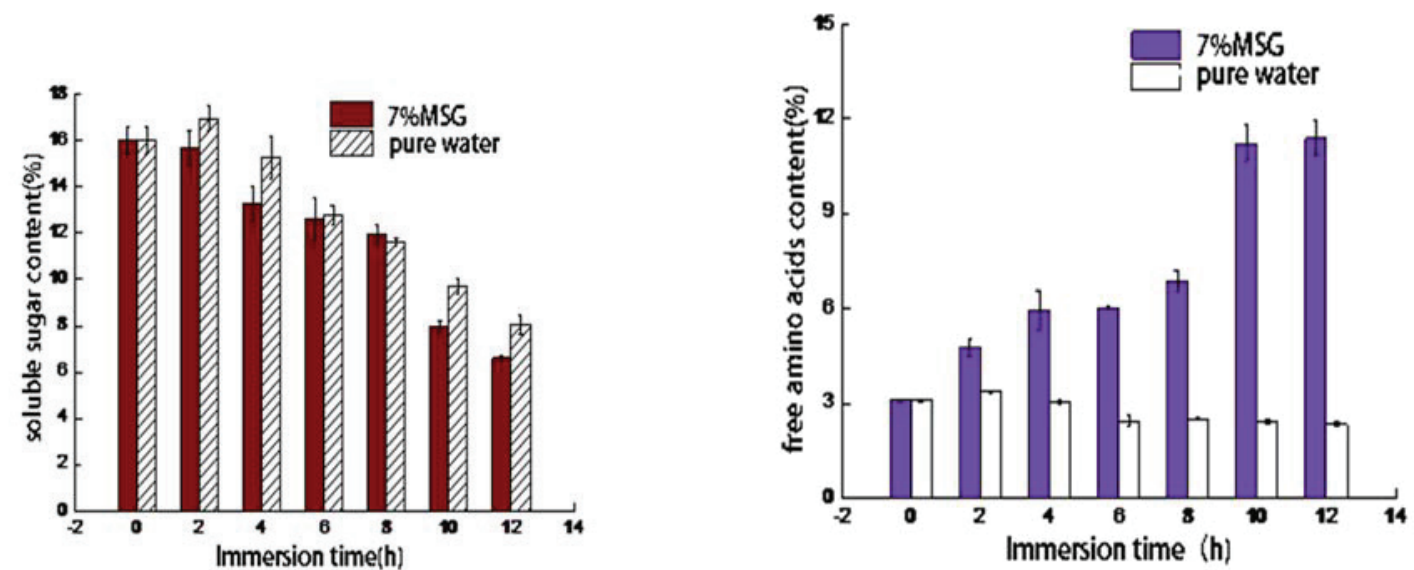

Fig.7 Effects of different immersion time on soluble sugar content of Moringa oleifera leaves Fig.8 Effects of different immersion time on amino acids content of Moringa oleifera leaves

The same immersion time, pure water treatment group soluble total sugar content higher than 7\% MSG solution treatment group is show in Fig.7. With the prolongation of immersion time, the content of soluble sugar in the two treatments showed a gradual decrease, which may be due to the oxidation and dissolution of the soluble sugar itself, and the activity of some enzymes under low oxygen condition was inhibited. And other substances difficult to hydrolysis reaction, thereby reducing the total amount of soluble sugar. With the prolongation of immersion time, the content of soluble sugar in the two treatments showed a gradual decrease, which may be due to the oxidation and dissolution of the soluble sugar itself, and the activity of some enzymes under low oxygen condition was inhibited. And other substances difficult to hydrolysis reaction, thereby reducing the total amount of soluble sugar.

With the prolongation of soaking time, CK treatment group increased first and then decreased, D treatment group showed a gradual upward trend, and tends to gentle (Fig.8), this may be related to Polyamine degradation produces GABA and beta-alanine [19]. Although The decrease in L-glutamate content is due to change to GABA, other amino acids was increased, and the total amount of free amino acids was increased. With the excessive accumulation of GABA, the GABA synthesis pathway was inhibited. GABA degrading enzyme is activated, so that the free amino acid content is reduced. After treatment with $2 \mathrm{H}$, the content of free amino acids in CK treatment group began to decrease, this may have a certain relationship with the D-treated group to promote the GABA synthesis substrate, resulting in a longer increase in GABA content.

\subsubsection{The effect of different immersion time on the} color and odor of Moringa oleifera leaves

Table.2 Effects of different treatment time on color and smell of fresh Moringa oleifera leaves

\begin{tabular}{|c|c|c|}
\hline Processing time $(\mathrm{h})$ & Pure water treatment group & $7 \%$ MSG treatment group \\
\hline 0 & Green leaves, rich fragrance & Green leaves, rich fragrance \\
\hline 2 & Leaves green, fragrant strong & Leaves green, fragrant strong \\
\hline 4 & Leaves green, fragrance weakened & Leaves green, fragrant strong \\
\hline 6 & Leaves green, appear qingqi & Leaves green, fragrant high \\
\hline 8 & $\begin{array}{l}\text { Leaf color dark, slightly water boring taste, } \\
\text { Qingqi obvious }\end{array}$ & Leaves green, fragrant high \\
\hline 10 & $\begin{array}{l}\text { The leaf crease is increased, the leaves become } \\
\text { soft, the water is stuffy, the taste is obvious, } \\
\text { and there is sour smell }\end{array}$ & Leaves green, slightly watery taste \\
\hline 12 & $\begin{array}{l}\text { Crease red, leaves are softer, water boring } \\
\text { serious, sour smell obvious }\end{array}$ & $\begin{array}{l}\text { Crease red, leaves are softer, water } \\
\text { boring serious, sour smell obvious }\end{array}$ \\
\hline
\end{tabular}

The color and odor of the fresh leaves of the two treatments changed with the immersion time are show in Tab.2. From $0 \sim 4 \mathrm{~h}$, the two groups of fresh leaves are green, fragrance is also more rich. When the pure water treatment group was treated for $6 \mathrm{~h}$, the fresh leaf smelled with qingqi gas. Since then, with the immersion time, leaf redness, crease increased, leaves become soft, water taste serious. 7\% MSG solution treatment group $6 \mathrm{~h}$ and $8 \mathrm{~h}$, the fresh leaves remain green, the fragrant is still obvious. Treatment $10 \mathrm{~h}$, the leaf color green, there is a slight water taste, but the overall effect of fresh leaves on the little impact.

\section{Conclusion}

There were significant differences in the quality of the three kinds of drying methods. In general, $60{ }^{\circ} \mathrm{C}$ blast drying method is the most suitable for industrial production of GABA Moringa oleifera leaves method. Under the 
condition of $60^{\circ} \mathrm{C}$ blast drying, the tea color brightness and soluble sugar content is higher, free amino acids was moderate, flavonoids was not the same as that of natural dry and vacuum freeze-drying. The difference of GABA content and vacuum freeze-drying was not significant. The use of $60^{\circ} \mathrm{C}$ blast drying to prepare Moringa oleifera leaves, the equipment requirements are low, drying time is short, free from weather constraints, easy to control. The highest GABA content in the Moringa oleifera leaves treated with $7 \%$ MSG solution for $10 \mathrm{~h}$, and the effect was the best. The content of flavonoids, polyphenols, soluble sugar and free amino acids in the leaves of Moringa oleifera was also ideal, and the color of the fresh leaves was green after the treatment,but within the acceptable range of sensory quality. So choose 7\% MSG solution and soak $10 \mathrm{~h}$ as the preparation of high content of GABA Moringa oleifera leaves .

In summary, the fresh Moringa oleifera leaves of soaked in $7 \% \mathrm{MSG}$ for $10 \mathrm{~h}$, using $60{ }^{\circ} \mathrm{C}$ blast drying treatment is a way to prepare high level of GABA Moringa oleifera leaves.

\section{Acknowledgments}

This research was supported by the Fundamental Scientific Research Funds for Chinese Academy of Tropical Agricultural Sciences (Project No. 1630122016007) and the Fundamental Scientific Research Funds for Chinese Academy of Tropical Agricultural Sciences (Project No. 1630122016011).

\section{References}

1. YUE Xiu-jie, LI Chao, FU Xiong.Preparation of Flavonoids from Moringa oleifera Leaves by Ultrasonic Wave and Its Antioxidant Activity [J]. food science and technology,2016, 37 (1).

2. Oluduro O A, Aderiye B I, Connolly J D, et al. Characterization and Antimicrobial Activity of 4(beta-D-Glucopyranosyl-1->4-alpha-Lrhamnopyranosyloxy)-benzyl thiocarboxamide; a Novel Bioactive Compound from Moringa oleifera Seed Extract[J]. Folia Microbiologica, 2010, 55(5):422-426.

3. Fatoba T A, Faleyimu O I, Adebayo A J. Effects of Increasing Aqueous Root Extract of Moringa oleifera on Sperm Production of Albino Rats[J]. Agrosearch, 2013, 13(1):29.

4. Ogbunugafor H, Igwoezikpe M, Igwilo I, et al. In vitro and in vivo evaluation of antioxidant properties of Moringa oleifera ethanolic leaves extract and effect on serum lipid indices in rat[J]. Macedonian Journal of Medical Sciences, 2013, 5(4):397-403.

5. Nwamarah, Joy Ugo, Otitoju, et al. Effects of Moringa oleifera Lam. aqueous leaf extracts on follicle stimulating hormone and serum cholesterol in Wistar rats[J]. African Journal of Biotechnology, 2015.

6. Jung I L. Soluble extract from Moringa oleifera leaves with a new anticancer activity[J]. Plos One, 2014, 9(4):95492.
7. AbdELA, El BBS, Mahboub H D, et al. Moringa oleifera leaf extract ameliorates alloxan-induced diabetes in rats by regeneration of $\beta$ cells and reduction of pyruvate carboxylase expression[J]. Biochemistry and cell biology, 2014, 92(5):413-9.

8. Tsuchiya K, Matsuda S, Hirakawa G, et al. GABA production from discolored laver by lactic acid fermentation and physiological function of fermented laver[J]. Food Preservation Science, 2011, 33(3):121125.

9. Diana M, Quílez J, Rafecas M. Gamma-aminobutyric acid as a bioactive compound in foods: a review $[\mathrm{J}]$. Journal of Functional Foods, 2014, 10(3):407-420.

10. Zhou X L, Zhao L, Technology A, et al. Physiological function of GABA and its application in food[J]. Food Industry, 2011.

11. Wang H F, Chuang S M, Hsiao C C, et al. A synergistic effect of GABA tea and copper(II) on DNA breakage in human peripheral lymphocytes[J]. Food \& Chemical Toxicology, 2011, 49(4):955-962.

12. Khwanchai P, Chinprahast N, Pichyangkura R, et al. Gamma-aminobutyric acid and glutamic acid contents, and the GAD activity in germinated brown rice (Oryza sativa L.): Effect of rice cultivars[J]. Food Science and Biotechnology, 2014, 23(2): 373-379.

13. Deewatthanawong, R, Nock, J. F, Watkins, C. B. $\gamma$ Aminobutyric acid (GABA) accumulation in four strawberry cultivars in response to elevated $\mathrm{CO} 2$ storage[J]. Postharvest Biology \& Technology, 2010, 57(2):92-96.

14. Thatchapol Chungcharoen, Somkiat Prachayawarakorn, Patcharee Tungtrakul, et al. Effects of Germination Process and Drying Temperature on Gamma-Aminobutyric Acid (GABA) and Starch Digestibility of Germinated Brown Rice[J]. Drying Technology, 2014, 32(6):742-753.

15. Vongsak B, Sithisarn P, Mangmool S, et al. Maximizing total phenolics, total flavonoids contents and antioxidant activity of Moringa oleifera, leaf extract by the appropriate extraction method[J]. Industrial Crops \& Products, 2013, 44(1):566-571.

16. Wei Wei. Preparation and quality of mulberry tea with high content of GABA [D]. Southwestern University, 2014.

17. Tuin L G, Shelp B J. In situ, L-Glutamate Metabolism by Developing Soybean Cotyledons I. Metabolic Routes[J]. Journal of Plant Physiology, 1994, 143(1):1-7.

18. Shelp B J, Bozzo G G, Trobacher C P, et al. Hypothesis/review: contribution of putrescine to 4aminobutyrate (GABA) production in response to abiotic stress[J]. Plant Science An International Journal of Experimental Plant Biology, 2012, 193194(193-194):130-5. 